\title{
A-06 \\ UMBRALES SOSTENIBLES DE ESTRÉS HÍDRICO DURANTE EL ENDURECIMIENTO DEL HUESO EN LA PRODUCCIÓN DE ACEITUNA DE MESA
}

Corell, $M$ (1), Martín-Palomo, MJ (2), Girón, I (3), Torrecillas, A (4), Pérez-López, D (5), Moreno, $F(6)$, Moriana, A (7) ${ }^{1}$ Profesora, Universidad de Sevilla, Departamento de Ciencias Agroforestales,
mcorell@us.es
$\begin{aligned} & { }^{2} \text { Profesora, Universidad de Sevilla, Departamento de Ciencias Agroforestales, } \\ & \text { mjpalomo@us.es }\end{aligned}$

3 Técnico superior, Consejo Superior de Investigaciones Científicas, IRNAS, iggi@irnase.csic.es

${ }^{4}$ Profesor de Investigación,Consejo Superior de Investigaciones Científicas, CEBAS, atorreci@cebas.csic.es

${ }^{5}$ Profesor, Universidad Politécnica de Madrid, Departamento de Producción Vegetal:Fitotecnia, david.perezl@upm.es

${ }^{6}$ Profesor de Investigación,Consejo Superior de Investigaciones Científicas, IRNAS, fmoreno@irnase.csic.es

${ }^{7}$ Profesor, Universidad de Sevilla, Departamento de Ciencias Agroforestales, amoriana@us.es

\section{Resumen}

La programación del riego deficitario controlado (RDC) es una necesidad derivada de la escasez de agua disponible para el riego. La resistencia al estrés hídrico de los diferentes estados fenológicos en olivar está bastante bien documentada pero no así los niveles de estrés hídrico. El objetivo de este trabajo es sugerir en base a experimentos realizados en los últimos 7 años los niveles de estrés hídrico para el manejo del RDC en olivar.Los experimentos descritos en la presente comunicación se han desarrollado en la finca "La Hampa" propiedad del IRNAS (CSIC) en Coria del Río (Sevilla), en un olivar de 37 años cV "Manzanillo" y a un marco de 7*5m. Los experimentos se establecieron con diferentes tratamientos de riego, aplicando condiciones de estrés hídrico durante endurecimiento de hueso y unas semanas antes de cosecha. Se discute los diferentes umbrales detectados y su efecto en cosecha, cantidad y calidad, y en la fisiología de la planta en el años en curso en el ciclo bianual del olivo.

\section{1.- Introducción}

El olivar es uno de los cultivos leñosos que ha sufrido un mayor cambio en las últimas dos décadas. Este cultivo ha pasado de ser tradicional de secano con una baja inversión, a un cultivo en el que aproximadamente un $20 \%$ de su superficie está en regadío (400.000 ha, Magrama, 2016) y con un aumento considerable en el número de árboles por hectárea y en la inversión realizada. Esta transformación supuso la aparición de un nuevo consumidor de agua sin apenas trabajos de investigación que permitan una optimización del riego.La programación de riego deficitario controlado (RDC) especialmente en cultivos leñosos es una necesidad derivada de la escasez de agua disponible para el riego. En el caso del olivar es aún más importante por la escasa dotación de agua y por su gran capacidad de resistencia al estrés hídrico. La resistencia al estrés hídrico de los diferentes estados fenológicos está bastante bien documentada en la mayor parte de los cultivos y el olivo no es una excepción (Goldhamer, 1999). Sin embargo, aunque en base a esta información se sugieren manejos, incluso para las nuevas plantaciones 
superintensivas(Fernández, 2014) se conoce muy poco acerca de los umbrales de estrés hídrico a los que puede someterse a la planta.EI RDC ha sido programado tradicionalmente en función de un porcentaje de la evaportanspiración del cultivo (ETc) en condiciones no limitantes. Sin embargo, la respuesta fisiológica de las plantas al estrés hídrico dificultad la modelización y estimación de la ETc. Desde los años 90 del pasado siglo se han desarrollado sensores que miden de forma continua diferentes indicadores de relaciones hídricas en las plantas. El desarrollo de la electrónica y las tecnologías de la información ha dado lugar a una gama cada vez más amplia de posibilidades. No obstante, a pesar de todo este espectacular avance, aún se sigue sin describir cuales son los límites a los que se puede someter a los árboles durante los periodos de déficit. El objetivo de este trabajo es sugerir en base a experimentos realizados en los últimos 7 años los niveles de estrés hídrico para el manejo del RDC en olivar.

\section{2.- Materiales y métodos}

Los experimentos se llevaron a acabo en la finca La Hampa propiedad del Instituto de Recursos Naturales y Agrobiología de Sevilla (IRNAS-CSIC) localizada en Coria del Río (Sevilla, España) $\left(37^{\circ} 17^{\prime} \mathrm{N}, 6^{\circ} 3^{\prime} \mathrm{W}, 30 \mathrm{~m}\right.$ altitud). Los experimentos se realizaron en un olivar de mesa (cv Manzanilla) de 37 años de edad al comienzo de los experimentos (año 2008) con un marco de $7 \mathrm{~m} \times 5 \mathrm{~m}$. El suelo es de tipo franco arenoso con unos $2 \mathrm{~m}$ de profundidad con un contenido en agua de $0.33 \mathrm{~m}^{3} \mathrm{~m}^{-3}$ a saturación, $0.21 \mathrm{~m}^{3} \mathrm{~m}^{-3}$ a capacidad de campo y $0.10 \mathrm{~m}^{3} \mathrm{~m}^{-3}$ en el punto de marchitez permanente. La densidad aparente es de $1.5 \mathrm{~g} \mathrm{~cm}^{-3}$. El riego se realiza habitualmente durante la noche o últimas hora de la tarde mediante el uso de goteo (5 emisores de $8 \mathrm{I} \mathrm{h}^{-1}$ por árbol) con una calidad de agua que no se considera limitante para el cultivo.

Los datos climatológicos se obtuvieron de una estación de una estación automática completa situada a $40 \mathrm{~m}$ de la parcela. La evapotranspiración de referencia (ETo) se calculó empleando la ecuación de Penman-Monteith (Allen et al., 1998).

El diseño experimental fue en bloques al azar con 3 o 4 bloques, dependiendo del periodo, y tres tratamientos diferentes de riego. Los tratamientos de riego tuvieron en consideración el estado fenológico del cultivo a la hora de aplicar las restricciones de riego. Las fases consideradas fueron:

- Fase I. Desde brotación hasta comienzo de endurecimiento del hueso. Esta fase engloba la plena floración (que suele tener lugar para el año medio a mediados de Abril) y es considerada la más sensible al estrés hídrico (Moriana et al., 2003).. El inicio de brotación habitualmente ocurre desde final de Febrero y el comienzo del endurecimiento masivo del hueso en la última semana de Junio. La determinación del comienzo del endurecimiento masivo se realizó según Rapoport et al (2013) que brevemente consiste en medir la longitud de la aceituna, cuando hay un cambio en la tasa de crecimiento se considera que ha comenzado este proceso. En el año medio durante este periodo las necesidades de riego suelen ser pequeñas por el régimen pluviométrico de la zona.

- Fase II. Desde endurecimiento masivo de hueso hasta la última semana de Agosto. Esta fase es la más resistente al estrés hídrico (Goldhamer, 1999) y es la que analizaremos en este trabajo. En todos los casos el final de esta fase es arbitrario y se ha seleccionado por la fecha de cosecha para asegurar un periodo de rehidratación suficiente. En el año medio esta fase coincide con una ausencia total de lluvias y una gran demanda evaporativa.

- Fase III. Desde la última semana de Agosto hasta cosecha. Esta fase se ha empleado como periodo de rehidratación habitualmente. La fecha media de cosecha es en torno al 15 de Septiembre. En el año medio puede iniciarse el periodo de lluvias otoñales aunque habitualmente de forma escasa.

- Postcosecha.

Los tratamientos realizados fueron: 
- Control. $100 \%$ de la Evapotranspiración del cultivo empelando para el cálculo los coeficientes de cultivo sugeridos por Fernández et al (2006) para la parcela ( $\mathrm{Kc}=0.7$ durante la fase de endurecimiento) y estimando un coeficiente reductor $(\mathrm{Kr})$ de 0.8. No se tuvo en consideración la humedad del suelo. Sin riego en postcosecha.

- Riego deficitario 2 (RDC-2). Restricción del riego durante la Fase II desde el año 2008 al 2013 estableciendo un umbral de riego alrededor a -2.5 MPa. El riego durante las Fases I y III se realizaba en función del umbral sugerido por Moriana et al (2012) de $-1.2 \mathrm{MPa}$ (en Fase I) y $-1.4 \mathrm{MPa}$ (en Fase II). Sin riego en postcosecha.

- Riego deficitario 12 (RDC-12). Restricción del riego en fase I y II desde el año 2008 al 2013 con umbrales similares al RDC-2.Sin riego en postcosecha.

- Riego deficitario controlado 2 semanas antes de cosecha (RDC- 2s). Riego como el control con un recorte de riego dos semanas antes de cosecha para obtener un nivel de estrés de -2.0 MPa. Sin rehidratación ni riego en postcosecha. Aplicado sólo en 2014.

- Riego deficitario controlado 4 semanas antes de cosecha (RDC-4s). Como el anterior pero cuatro semanas antes de cosecha.

La aplicación del agua de riego en los tratamientos deficitarios fueron diarios cuando era preciso regary la estimación de las necesidades fue proporcional a la distancia de los valores medidos en planta al umbral considerado (Moriana et al 2012; Girón et al 2015). No realizando ningún riego cuando las plantas estaban mejor hidratadas que los umbrales fijados.

El estado hídrico de las plantas se determinó mediante la medida de potencial hídrico, conductancia estomática y dendrometría. Las medidas de potencial hídrico se realizaron al mediodía en hoja cubierta al menos una hora antes realizado con una cámara de presión (100, PMS). Las medidas de conductancia estomática se realizaron sobre las 10:00 am para estimar el máximo diario con un porómetro de régimen permanente (LICOR1600). Las medidas de dendrometría se realizaron con sensores de desplazamiento (LVDT, DF2.5, Solartrón) que obtenían datos cada 30 segundo y medias cada 15 minutos. El parámetro empleando en este trabajo es la tasa de crecimiento del tronco que se calcula como la diferencia entre dos máximos diarios consecutivos.

La cosecha se realizó de forma individual por cada árbol experimental estimando mediante muestreo el número de frutos por Kilo (escandallo) y la relación pulpa hueso.

\section{3- Resultados y discusión}

Los diferentes tratamientos de riego realizados entre los años 2008 al 2013 dieron lugar a niveles de estrés hídrico diferentes influenciados por la carga de frutapero sobre todo por la pluviometría. La Figura 1, muestra, a modo de ejemplo, dos años con un nivel de carga record y resultados muy distintos en cuanto al estado hídrico fruto de la abundancia de lluvias primaverales. En ambos casos la estrategia era de recorte durante el momento de endurecimiento del hueso y en ninguno de los casos se regó en ese periodo (entre las líneas verticales en la figura 1). El nivel alcanzado en el caso del año 2008 (Fig 1a) fue de un potencial hídrico mínimo alrededor de $-3 \mathrm{MPa}$, con una rehidratación casi completa al final de la estación. Por el contrario, en el año 2010 (Fig. 1b) el máximo nivel de estrés hídrico apenas si llegó a -2 MPa. Es importante reseñar que en ambos casos los árboles Control, sin carencia de agua, llegaron hasta valores alrededor de -2 MPa. Este comportamiento está probablemente relacionado con la presencia del fruto que da lugar a una mayor traspiración de la planta (Martin-Vertedor et al., 2011).

La Figura 2 presenta los datos de conductancia estomática máxima diaria en los mismos años presentados en la Figura 1. Los niveles de potencial hídrico dieron lugar a un efecto en el intercambio gaseoso. Niveles de potencial por debajo de -2 MPa dieron lugar a un cierre estomático significativo en los árboles RDC (Fig 2a), mientras que los niveles de potencial por encima mantuvieron valores de conductancia foliar similares al control durante 
toda la estación (Fig. 2b). Moriana y Fereres (2002) describieron el intercambio gaseoso como un proceso menos sensible al estrés hídrico que el potencial hídrico. La no afección de la apertura estomática con valores superiores a -2 MPasugieren un primer nivel de estrés hídrico que podríamos catalogar como bajo, mientras que valores inferiores podrían ser moderados. Moriana et al (2002) presentan reducciones mucho más severas de la conductancia foliar con niveles de potencial hídrico por debajo de los $-3 \mathrm{MPa}$, lo que ya podría sugerir un nivel severo, al menos desde el punto de vista del estado hídrico del árbol.
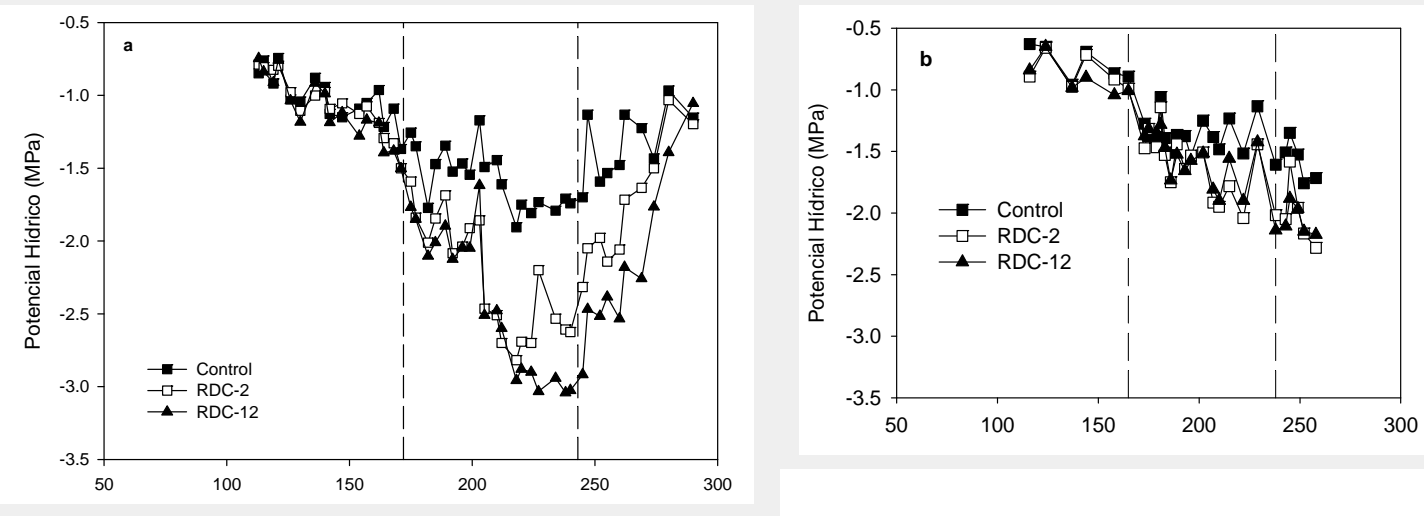

Figura 1. Evolución del potencial hídrico durante los años 2008 (a) y 2010 (b) con niveles de carga record para la media de la parcela (16.6 T ha ${ }^{-1}$ en 2008 y $16 \mathrm{~T} \mathrm{ha}^{-1}$ en 2010). Cada punto es la media de 6 árboles. Las líneas verticales indican el periodo de endurecimiento masivo del hueso, momento en el que se aplicó condiciones de RDC (Riego deficitario Controlado).

Estos niveles de estrés deben ser relacionados con el efecto en cosecha antes de considerarlos en la programación del riego. El efecto del estrés hídrico sobre la cosecha puede producirse a dos escalas, en el año en curso, afectando al número o tamaño de los frutos, y en la cosecha siguiente, disminuyendo el crecimiento o reducción el número de yemas inducidas a flor.
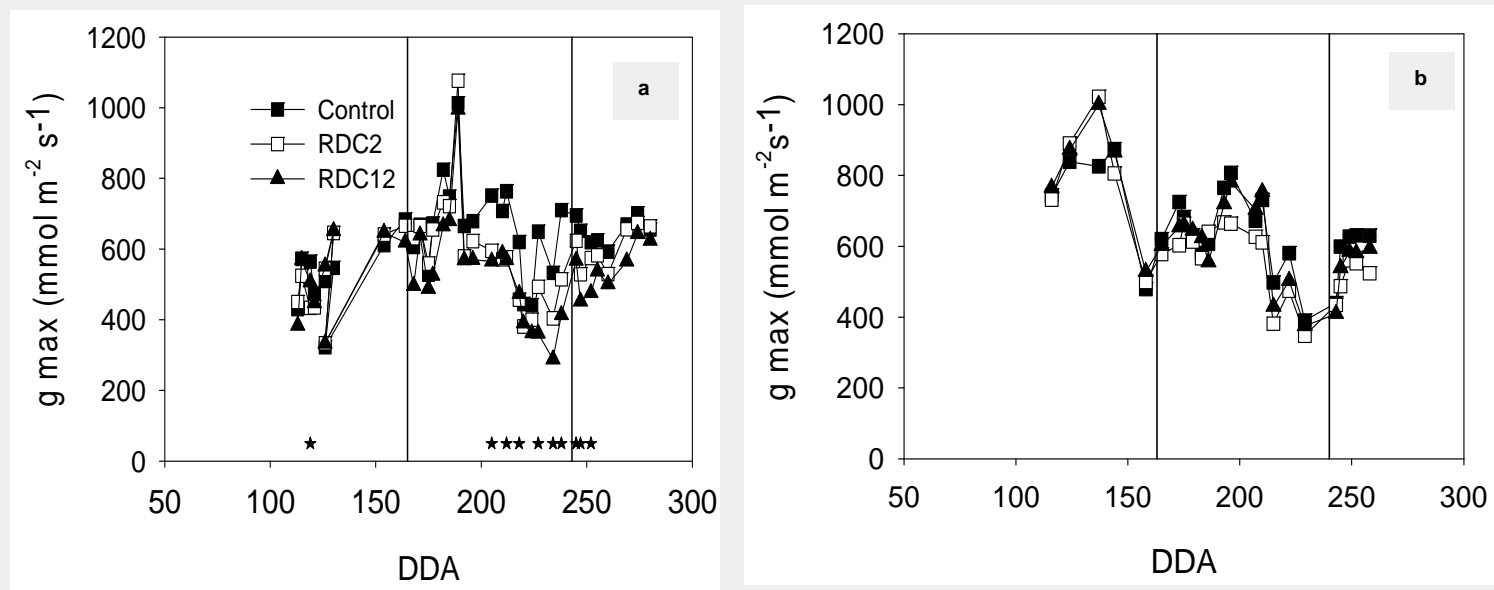

Figura 2. Evolución de la conductancia foliar máxima durante los años 2008 (a) y 2010 (b)con niveles de carga record para la media de la parcela (16.6 T ha ${ }^{-1}$ en 2008 y $16 \mathrm{~T}^{\mathrm{T}} \mathrm{ha}^{-1}$ en 2010). Cada punto es la media de 6 árboles. Las líneas verticales indican el periodo de endurecimiento masivo del hueso, momento en el que se aplicó condiciones de RDC (Riego deficitario Controlado). Los asteriscos indican diferencias significativas en esa fecha $(p<0.05$. Test Tukey). 
La Figura 3a presenta la evolución del volumen de fruto en el año 2012, un año de carga media (alrededor de $6 \mathrm{~T} / \mathrm{ha}$ ), y nivel de estrés moderado con valores de potencial hídrico por debajo de $-2 \mathrm{MPa}$ al final de endurecimiento de hueso. Las diferencias significativas en el crecimiento del fruto sólo tuvieron lugar durante este periodo final en el que se detectó una disminución del potencial. El periodo de rehidratación, alrededor de 3 semanas, perimitó una recuperación completa tanto del estado hídrico como del tamaño del fruto. La Figura 3b presenta los datos de volumen de fruto en el año 2014, un año record de carga (alrededor de $15 \mathrm{~T} / \mathrm{ha}$ ) y en el que sólo se suprimió el riego unas semanas antes de cosechar sin rehidratar. Los valores de potencial hídrico estuvieron ligeramente por debajo de -2 MPa.No hubo diferencias significativas en el tamaño del fruto al final del experimento aunque se aprecia una parada de los tratamientos deficitarios que coincide con una tendencia, no significativa, a tener un mayor número de frutos por Kilo (datos no mostrados).

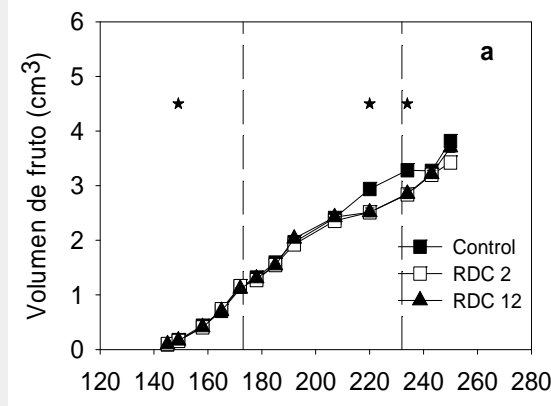

DDA

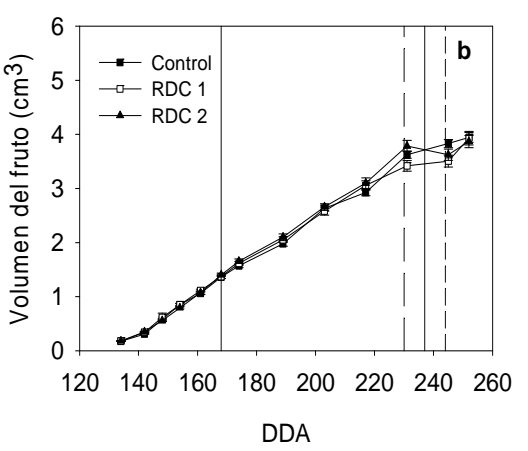

DDA

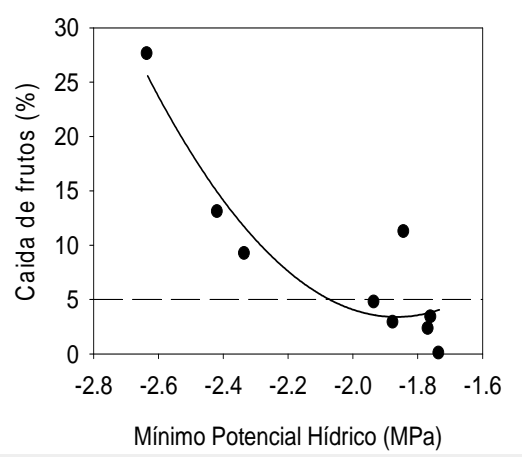

Figura 3. Evolución del volumen de fruto en el año 2012 (a) y 2014 (b). La figura 3c representa la relación entre la caid de frutos en brotes marcados y el potencial hídrico mínimo medido durante el endurecimiento del hueso entre los años 2011 al 2013. Cada punto es la media de 6 árboles. Las líneas verticales indican el periodo de endurecimiento masivo del hueso (a) o del recorte de agua antes de cosecha (b). La línea horizonatal de la figura $3 c$ indica un nivel asumible de caída de frutos. Los asteriscos indican diferencias significativas en esa fecha ( $p<0.05$. Test Tukey).

La Figura 3c presenta la relación entre el número de frutos caídos entre los años 2011 al 2013 y el potencial mínimo obtenido durante la fase de endurecimiento de hueso. Los datos indican una clara tendencia a incrementar la reducción de frutos con la disminución del potencial hídrico. Asumiendo una caída del 5\% como económicamente viable, de nuevo, los niveles de estrés hídrico en torno a -2 MPa podrían ser adecuados durante este estado fenológico. A pesar de esta reducción tan clara en el número de frutos con el nivel de estrés (niveles que llegan hasta el 25\% de los frutos por brote) está disminución no se vio trasladada en el mismo orden de magnitud en la cosecha obtenida (datos no mostrados). Esta falta de relación lineal podría estar relacionada con un efecto de muestreo y una caída diferencial dependiendo de la zona del árbol. Cherbity-Hoffman et al (2012) describe una distribución de la cosecha en los setos de olivar dependiente de la radiación que reciban.

La Figura 4 presenta los datos de la evolución de los diámetros máximos en dos años diferentes 2009 (año de descarga, Figura 4a) y 2013 (año de carga, Figura 4b). Durante el año 2009, los valores de potencial hídrico durante el endurecimiento de hueso disminuyeron hasta valores cercanos a $-2.5 \mathrm{MPa}$ lo que dio lugar, incluso a niveles de estrés hídrico inferiores, a una parada del crecimiento en los árboles RDC respecto al control. La rehidratación de los árboles permitió al final del crecimiento una tasa de crecimiento del tronco de nuevo cercana al control. Esta restricción del crecimiento tan evidente no afectó a la coseca del año siguiente en la que se obtuvieron valores del mismo orden que el control (15 T/ha en Control vs 19.2 T/ha en RDC, Moriana et al 2013). En los años en carga, el crecimiento se detiene durante la fase de endurecimiento (año 2013, Figura 4) por efecto de 
la mayor demanda de asimilados del fruto, por lo que el efecto en la cosecha está en el diferencial de crecimiento que tenga lugar durante la fase de brotación-floración (Fase I) en la que el fruto no actúa como limitante. En los datos mostrados en la figura los valores de potencial hídrico fueron similares y es necesario indicadores más precisos para controlar esta fase (pej. la variación del diámetro del tronco, Moriana y Fereres, 2002).
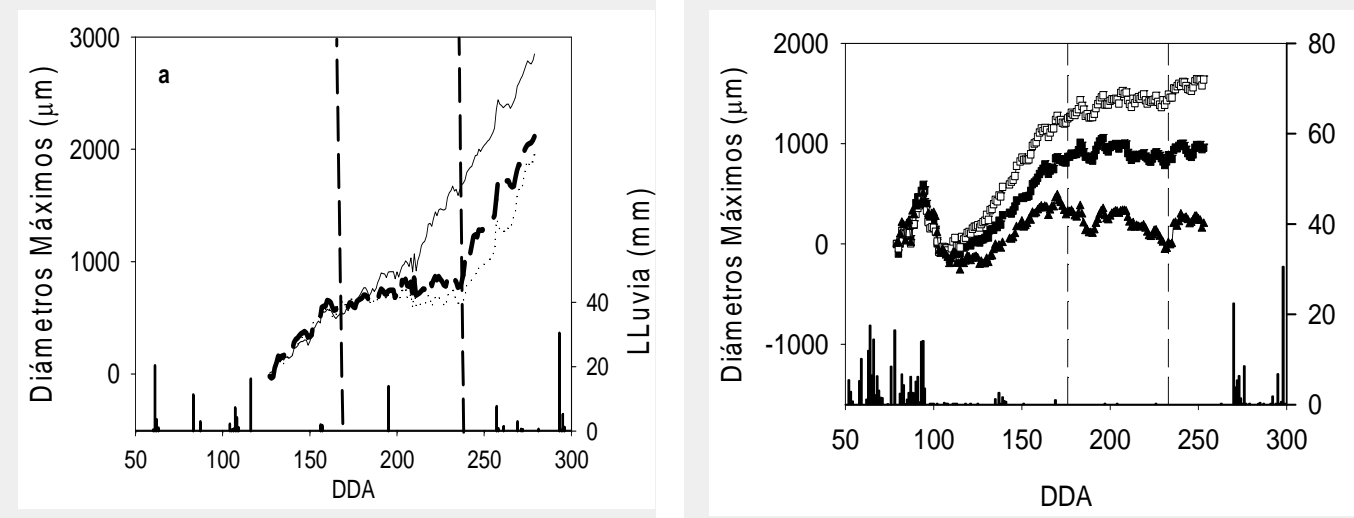

Figura 4. Evolución de los diámetros máximos durante un año de descarga (2009, a) y uno de carga $(2013$, b). La línea continua representa el tratamiento control y la discontinua los tratamientos deficitarios (Figura a). Los cuadrados rellenos representan el tratamiento control y los triángulos y cuadrados vacíos los tratamientos deficitarios (Figura b). En la parte inferior de la figura se representan la precipitación. Las líneas verticales indican la fase de endurecimiento del hueso.

La inducción floral del año siguiente es otro proceso que podría estar alterado con condiciones de estrés hídrico durante la fase de endurecimiento ya que algunos autores sugieren que el comienzo tiene lugar en esta época del año. Los experimentos realizados desde el año 2008 al 2013 muestran que el número de inflorescencias en el momento previo a plena floración nunca han estado afectadas. La evolución típica es la que se muestra en la Figura 5 correspondiente al año 2013 y en la que ya se ha descrito un estrés en el verano previo con niveles de $-2.5 \mathrm{MPa}$. Los valores obtenidos al inicio y al final de la caída por cuajado son muy similares en todos los tratamientos lo que sugiere que no hubo un efecto del estrés del año anterior.

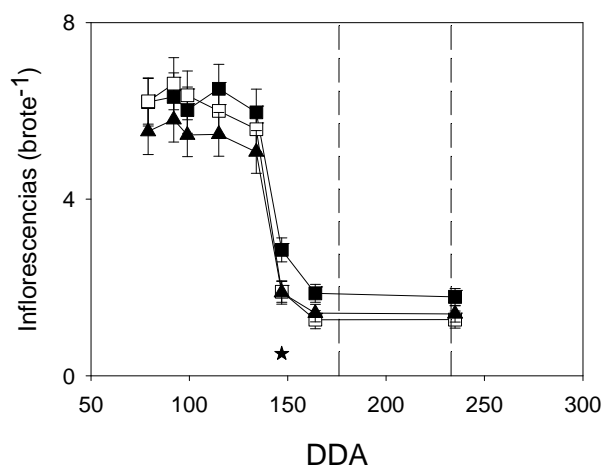

Figura 5. Evolución del número de inflorescencias durante el año 2013. Los cuadrados rellenos representan los datos Control, los cuadrados vacíos (RDC-2) y los triángulos (RDC12) los tratamientos deficitarios. Cada símbolo es la media de 60 datos. Las líneas verticales indican el periodo de endurecimiento del hueso.

La floración es un proceso muy sensible durante el año en curso (Moriana et al., 2003), aunque dado el régimen de lluvias de nuestras zonas olivareras no es habitual que tenga 
problemas. La inducción es un proceso poco conocido en su relación con el estrés hídrico pero no parece que los niveles a los que ha sido sometido vayan a comprometer la cosecha.

La Tabla 1 presenta los resultados en cantidad y calidad de cosecha del año 2008 al 2014. Nunca se obtuvieron diferencias significativas en cantidad de cosecha, aunque en muchas campañas (5 de las 7 presentadas) la cantidad de fruta fue superior a la de los tratamientos deficitarios.

Tabla 1.Cantidad y calidad en la cosecha del año 2008 al 2014. Letras diferentes en el mismo año indican diferencias significativas $(p<0.05$, Test de Tukey). Cosecha, T/ha; Escandallo, $\mathrm{n}^{\circ}$ frutos/ Kilo; Relación pulpa hueso; Riego, mm.

\begin{tabular}{|c|c|c|c|c|c|}
\hline Año & Tratamiento & Cosecha & Escandallo & Pulpa/Hueso & Riego \\
\hline $\mathbf{2 0 0 8}$ & Control & $18.3 \pm 0.31$ & $308 \pm 14$ & $5.4 \pm 0.2$ & 619 \\
\hline & RDC-2 & $16.8 \pm 1.42$ & $379 \pm 29$ & $4.5 \pm 0.4$ & 119 \\
\hline & RDC-12 & $14.6 \pm 2.5$ & $358 \pm 22$ & $4.4 \pm 0.4$ & 45 \\
\hline $\mathbf{2 0 0 9}$ & Control & $2.3 \pm 0.51$ & $229 \pm 14$ & $5.7 \pm 0.2 \mathrm{ab}$ & 585 \\
\hline & RDC-2 & $1.4 \pm 0.52$ & $225 \pm 10$ & $4.2 \pm 0.6 \mathrm{~b}$ & 110 \\
\hline & RDC-12 & $0.8 \pm 0.17$ & $228 \pm 3$ & $6.0 \pm 0.3 \mathrm{a}$ & 63 \\
\hline $\mathbf{2 0 1 0}$ & Control & $15.0 \pm 1.7$ & $281 \pm 31$ & $4.8 \pm 0.14 \mathrm{a}$ & 710 \\
\hline & RDC-2 & $19.2 \pm 2.9$ & $284 \pm 5$ & $4.0 \pm 0.12 \mathrm{~b}$ & 160 \\
\hline & RDC-12 & $13.9 \pm 1.3$ & $288 \pm 11$ & $4.2 \pm 0.11 \mathrm{ab}$ & 38 \\
\hline $\mathbf{2 0 1 1}$ & Control & $2.5 \pm 0.5$ & $188 \pm 5 \mathrm{~b}$ & $6.2 \pm 0.1 \mathrm{a}$ & 299 \\
\hline & RDC-2 & $4.1 \pm 0.6$ & $206 \pm 5 \mathrm{a}$ & $5.7 \pm 0.1 \mathrm{~b}$ & 145 \\
\hline & RDC-12 & $2.9 \pm 0.6$ & $190 \pm 4 \mathrm{ab}$ & $6.1 \pm 0.1 \mathrm{a}$ & 115 \\
\hline $\mathbf{2 0 1 2}$ & Control & $6.6 \pm 0.7$ & $233 \pm 13$ & $4.1 \pm 0.1$ & 540 \\
\hline & RDC-2 & $5.0 \pm 0.8$ & $249 \pm 10$ & $4.1 \pm 0.2$ & 171 \\
\hline & RDC-12 & $5.9 \pm 0.7$ & $240 \pm 10$ & $4.4 \pm 0.2$ & 161 \\
\hline $\mathbf{2 0 1 3}$ & Control & $9.0 \pm 1.1$ & $229 \pm 13$ & $4.6 \pm 0.3$ & 369 \\
\hline & RDC-2 & $8.2 \pm 0.6$ & $209 \pm 7$ & $5.2 \pm 0.2$ & 207 \\
\hline & RDC-12 & $6.7 \pm 0.7$ & $208 \pm 11$ & $5.0 \pm 0.3$ & 106 \\
\hline $\mathbf{2 0 1 4}$ & Control & $14.7 \pm 1.6$ & $244 \pm 9$ & $4.6 \pm 0.1$ & 278 \\
\hline & RDC-2s & $12.2 \pm 2.4$ & $261 \pm 21$ & $4.6 \pm 0.2$ & 242 \\
\hline & RDC-4s & $12.0 \pm 2.4$ & $275 \pm 15$ & $4.4 \pm 0.2$ & 143 \\
\hline
\end{tabular}

Estas diferencias son más acusadas con el tratamiento RDC-12 y podrían estar relacionadas con el crecimiento de los árboles dando lugar a árboles Control con mayor volumen de copa. El manejo comercial de la poda es muy probable que elimine estas diferencias. Las tendencias estacionales presentan ciclos veceros en todos los tratamientos con una intensidad muy similar lo que de nuevo sugiere que no hay un efecto del estrés hídrico al largo plazo. Tampoco hay diferencias significativas en el número de frutos por kilo (escandallo), aunque de nuevo también hay una tendencia a mayores frutos (en 5 de los 7 años) en los árboles Control que en cualquiera de las estrategias RDC. No obstante, las diferencias no son superiores a un $15 \%$ obteniéndose habitualmente en todos los tratamientos valores en torno a 250 frutos por Kilo que son considerados de buena calidad. La relación pulpa hueso es un índice de calidad de gran importancia en la aceituna de mesa. En este parámetro sí hay, en algunos años, diferencias significativas (en 3 de 7) pero son entre el tratamiento Control y el deficitario que ha visto restringido su riego durante la fase de endurecimiento. Con los datos disponibles no hay una relación clara con los indicadores de estrés hídrico. La ausencia de diferencias con el tratamiento que, teóricamente, sufrió condiciones de estrés hídrico durante el cuajado podrían sugerir una disminución del tamaño del hueso que compensa la disminución en pulpa posterior. Sin embargo, las mediciones en hueso tampoco han dado diferencias significativas (datos no mostrados). Cuando el estrés 
hídrico se redujo en el año 2014 al último periodo previo a cosecha no hubo diferencias entre tratamientos aunque los niveles de estrés hídrico en el tratamiento de RDC 4s estuvieron ligeramente por debajo de -2.0 MPa. Finalmente lo que se ha obtenido es un ahorro de agua considerable respecto a los árboles Control. Las dotaciones de los tratamientos RDC estuvieron alrededor de las permitidas por las confederaciones hidrográficas para este cultivo, incluso en algunos años claramente por debajo. Es de destacar que la cantidad de agua empleada en los RDC fue muy variable y estuvo ligada al manejo del agua en función de los umbrales de estrés hídrico marcados.

\section{4.- Conclusiones y recomendaciones}

Un nivel de estrés hídrico superior a -2.0 MPa de potencial hídrico de tronco medido al mediodía durante la fase de endurecimiento del hueso no supuso una alteración de la cosecha del año en curso. Valores inferiores hasta niveles alrededor de -3.0 MPa afectaron al crecimiento del fruto pero el periodo de rehidratación fue suficiente para no detectar pérdidas significativas en el tamaño del fruto. Estos niveles de estrés hídrico, tras 7 años de experimentos, tampoco afectaron a la cosecha a medio plazo. Solamente se detectó una tenencia a perder cosecha en tratamientos en los que se restringió el riego durante cuajado por efecto, probablemente, de un menor tamaño de árboles. Sin embargo, es probable que el manejo de la poda en la explotación compense esta disminución. Estos dos umbrales de estrés pueden ser valores muy útiles para el manejo del riego deficitario en olivar de mesa que aseguren un uso sostenibles de los recursos con niveles de cosecha adecuados para el agricultor.

\section{5.- Agradecimientos}

Los datos presentados en este trabajo han sido financiados por sucesivos proyectos del MINNECO (AGL2007-66279-C03-00; AGL2010-19201-CO4-03; AGL2013-45922-C2-1R).Los autores agradecen la colaboración de todos los alumnos proyecto fin de carreara que han colaborado en este periodo y las ayudas de D. Antonio Montero y Pepe Rodriguez, técnicos del CSIC por su inestimable ayuda. Este trabajo se ha desarrollado gracias a la Unidad Asociada CSIC-US "Uso sostenible de suelo y agua en la agricultura".

\section{6.- Referencias}

Allen, R.G., Pereira, L.S., Raes, D., Smith. M., (1998).Crop evaportranspiration.Guideline for computing crop water requirements. FAO irrigation and drainage paper $n^{\circ} 56$. Roma. FAO.

Cherbiy-Hoffmann, S.U., Searles, P.S., Hall, A.J., Rousseaux, M.C. (2012). Influence of light environment on yield determinants and components in large olive hedgerows following mechanical pruning in the subtropics of Southern Hemisphere. ScientiaHorticulturae 137, 36-42.

Fernández, J. E. (2014). Understanding olive adaptation to abiotic stresses as a tool to increase crop performance.Environ.Exp. Bot. 103:158-179.

Fernández, J. E., Díaz-Espejo, A., Infante, J. M., Duran, P., Palomo, M. J., Chamorro, V., Giron, I. F., Villagarcía, L., (2006). Water relations and gas exchange in olive trees under regulated deficit irrigation and partial root zone drying. Plant Soil 284, 273-291.

Girón, I.F., Corell, M., Martín-Palomo, M.J., Galindo, A., Torrecillas, A., Moreno, F., Moriana, A. (2015). Feasibility of trunk diameter fluctuations in the scheduling of regulated deficit irrigation for table olive trees without references trees. Agricultural Water Management 161:114-126Goldhamer, D.A., 1999. Regulated deficit irrigation for California canning olives.ActaHortic. 474, 369-372.

Martín-Vertedor, A.I., Pérez-Rodríguez, J.M., Prieto, H., Fereres, E., (2011). Interactive responses to water deficits and crop load in olive (Oleaeuropaea L., cv. Morisca).Water use, fruit and oil yield. Agric. Water Manage. 98, 941-949.

Ministerio de Agricultura, Alimentación y Medio Ambiente (MAGRAMA) (2016). Anuario de estadísticaagraria.http://www.magrama.gob.es/es/estadistica/temas/publicaciones/anu ario-de-estadistica/2014/default.aspx 
Moriana, A. y Fereres, E.(2002). Plant Indicators for Scheduling Irrigation for Young Olive Trees.IrrigationScience 21,83-90.

Moriana, A; Villalobos, F.J.; Fereres, E. (2002). Stomatal and photosynthetic responses of olive (Oleaeuropaea L.) leaves to water deficits. Plant, Cell and Environment 25:395405

Moriana, A., Orgaz, F., Fereres, E., Pastor, M. (2003). Yield responses of a mature olive orchard to water deficits. J. Amer. Soc. Hort. Sci. 128,425-431.

Moriana, A., Pérez-López, D., Prieto, M.H., Ramírez-Santa-Pau, M., Pérez-Rodriguez, J.M. (2012). Midday stem water potential as a useful tool for estimating irrigation requirements in olive trees.AgriculturalWater Management 112:43-54

Rapoport, H.F., Pérez-López, D., Hammami, S.B.M., Aguera, J., Moriana, A. (2013). Fruit pit hardening: physical measurements during olive growth. Annals Applied Biology 163, 200-208. 